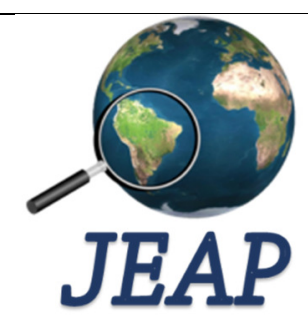

ISSN: 2525-815X

\section{Journal of Environmental} Analysis and Progress

Journal homepage: www.jeap.ufrpe.br/

\title{
Variabilidade de ondas de calor e a relação com o ENOS Modoki e Canônico em Santa Catarina
}

\section{Variability of heat wave and the relationship with ENSO Modoki and Canonical in Santa Catarina}

\author{
Rosandro Boligon Minuzzia ${ }^{\mathrm{a}}$ Carolina do Amaral Frederico ${ }^{\mathrm{a}}$ \\ ${ }^{a}$ Universidade Federal de Santa Catarina-UFSC, Centro de Ciências Agrárias, Av. Admar Gonzaga, n. 1356, Itacorubi, \\ Florianópolis-SC, Brasil. CEP: 88034-001. E-mail: rosandro.minuzzi@ ufsc.br; carols.ponqui@gmail.com.
}

\begin{tabular}{l}
\hline A R T I C L E I N F O \\
\hline Recebido 27 Jun 2017 \\
Aceito 11 Ago 2017 \\
Publicado 15 Ago 2017
\end{tabular}

\begin{abstract}
A B S T R A C T
Daily data of apparent temperature of four localities in Santa Catarina were used to analyze the spatial and temporal climatic variability of heat waves (HW) and their relationships with El Niño (EN) and La Niña (LN) Modoki and Canonical. A heat wave was defined as the event with at least three consecutive days of apparent temperature equal to or greater than the 90th percentile ("hot day") of each seasonal period analyzed (summer, fall, winter and spring). The number of occurrences and duration of local HW (LHW) and regional (RHW) were analyzed in sequence for the years of the historical series of each locality under study and divided into groups of years according to the occurrences of El Niño and La Niña Modoki and Canonical. The analyzes with the El Niño Southern Oscillation involved the spring from the start year (year 0 ) to the fall of the year of dissipation $($ year +1$)$ of the climatic driving forces. In each seasonal period, the number of "hot days" during LHWs last on average from 6 to 10 days. Only in Lages there has been a significant trend of increase in the last decades, the number of occurrences and the duration of the total of "hot days" during the LHW. The RHW increased only in the summer of the analyzed seasonal periods. El Niño and La Niña Modoki or Canonical do not influence the duration of 'hot days' during heat waves, except in Lages during La Nina Modoki events.
\end{abstract}

Keywords: Extreme climatic, El Niño, La Niña, maximum temperature.

\section{R E S U M O}

Dados diários de temperatura aparente de quatro localidades em Santa Catarina foram utilizados para analisar a variabilidade climática espacial e temporal de ondas de calor (OC) e suas relações com o El Niño (EN) e La Niña (LN) Modoki e Canônico. Definiu-se uma onda de calor, o evento com pelo menos três dias seguidos de temperatura aparente igual ou superior ao percentil 90 (“dia de calor") de cada período sazonal analisado (verão, outono, inverno e primavera). O número de ocorrências e a duração de OC local (OCL) e regional (OCR) foram analisadas em sequência para os anos da série histórica de cada localidade em estudo e divididas em grupos de anos, de acordo com as ocorrências dos eventos El Niño e La Niña Modoki e Canônico. As análises com o El Niño Oscilação Sul envolveram a primavera do ano de início (ano 0) até o outono do ano de dissipação $($ ano +1$)$ das forçantes climáticas. Para cada período sazonal, o número de "dias de calor" durante as OCL dura, em média, de 6 a 10 dias. Apenas em Lages houve tendência significativa de aumento, nas últimas décadas, no número de ocorrências e na duração do total de "dias de calor" durante as OCL. As OCR mostraram aumento apenas no verão para os períodos sazonais analisados. El Niño e La Niña Modoki 
ou Canônico não influenciam na duração de "dias de calor" durante ondas de calor, exceto em Lages durante eventos de La Niña Modoki.

Palavras-Chave: Extremo climático, El Niño, La Niña, temperatura máxima.

\section{Introdução}

Eventos extremos associados a altas temperaturas produzem impactos na saúde e mortalidade humana e, mesmo que em alguns casos não existam riscos de morte, trazem distúrbios sociais, econômicos e a ecossistemas. Diversos estudos projetam que os impactos na saúde causados por ondas de calor (OC) podem aumentar sob diversos cenários das mudanças climáticas, especialmente em países com baixa capacidade de se adaptar Diffenbaugh et al. (2005) notaram forte tendência positiva projetada na frequência e duração de eventos extremos de calor para o século 21.

Assim, o cenário futuro para a sociedade, economia e ecossistemas torna-se preocupante, como para os Estados Unidos, em que Ross \& Lott (2003) atribuíram as ondas de calor ocorridas no período de 1980 a 2003, por prejuízos industriais e agrícolas na ordem de bilhões de dólares por evento no país.

A situação geográfica da região sul do Brasil, garante a maior amplitude térmica do ciclo anual de temperatura no país, com o maior contraste entre o inverno e o verão (em torno de $11^{\circ} \mathrm{C}$ ). Além das variabilidades espacial e sazonal do clima médio na região, há também variabilidade climática em torno desse estado médio, em várias escalas de tempo. Por exemplo, há significativas oscilações interanuais de chuva e temperatura, em razão do fenômeno climático El Niño e La Niña (Grimm, 2009).

Recentemente, Ashok et al. (2007) apresentaram um diferente tipo de El Niño (EN) chamado de EN Modoki. Este tipo de EN ocorre no Pacífico Central, enquanto o EN Canônico (ou Convencional) ocorre no Pacífico Leste. Estes dois tipos também se aplicam para a La Niña (LN), apenas diferindo as características quanto as anomalias da temperatura superficial. Os impactos do EN Modoki no clima tropical e de latitudes divergem em relação ao EN Canônico devido a diferente intensidade e localização das anomalias positivas da TSM do Pacífico equatorial (Larkin \& Harrison, 2005; Ashok et al., 2007).

Ciente da sensibilidade que ecossistemas e vários setores de atividades humanas possuem em relação às adversidades climáticas, como ondas de calor e da influência do EN e da LN no clima, este estudo teve como objetivo analisar a variabilidade climática espacial e temporal de ondas de calor e suas relações com o ENOS Modoki e Canônico.

\section{Material e Métodos}

O estudo foi realizado em municípios de Santa Catarina: Campos Novos, Chapecó, Indaial e Lages.

Foram utilizados dados diários de temperatura média (T) e máxima do ar $\left(\mathrm{T}_{\text {máx }}\right)$ e umidade relativa do ar (UR), obtidos de estações meteorológicas pertencentes ao Instituto Nacional de Meteorologia (INMET). O período das séries históricas e os municípios (Figura 1) onde localizam-se as estações utilizadas no estudo, com suas respectivas coordenadas geográficas, altitude e o percentil 90 da temperatura aparente sazonal, constam na Tabela 1 .

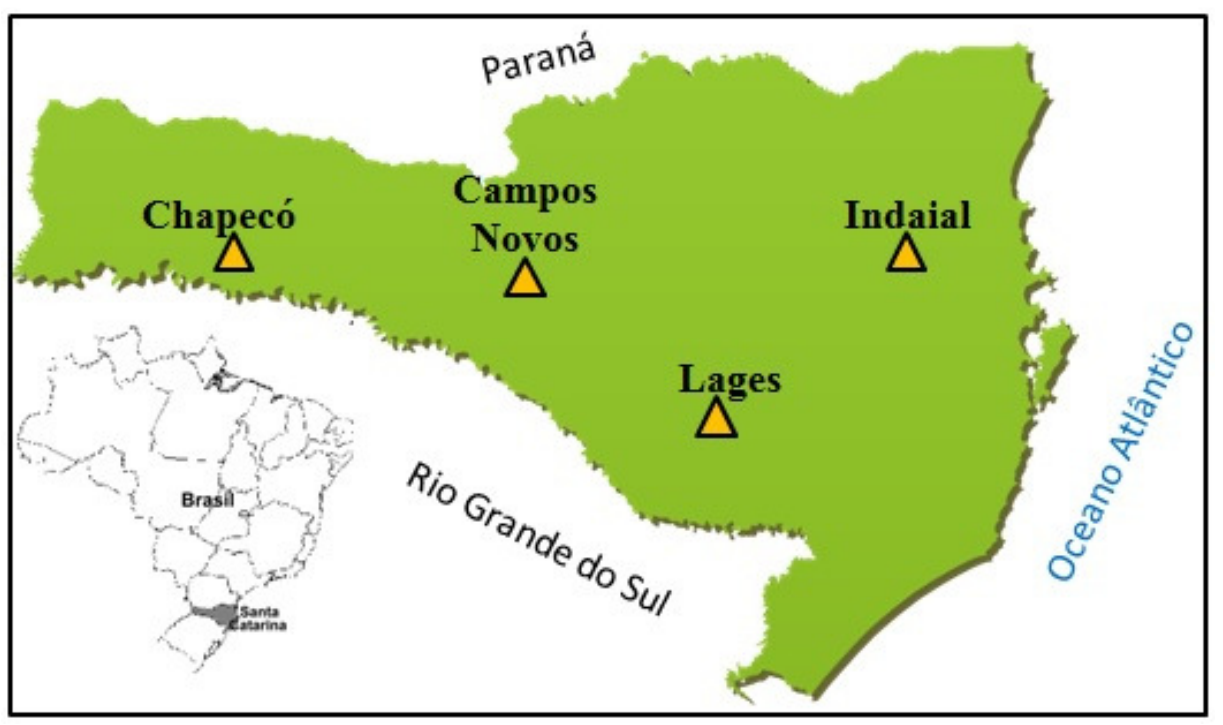

Figura 1. Localização geográfica dos municípios de Santa Catarina onde estão estabelecidas as estações meteorológicas utilizadas no estudo. 
Dentre vários critérios utilizados para definição de ondas de calor (OC), considerou-se como um evento de três ou mais 'dias de calor' consecutivos (Unal et al., 2012). Foi definido como um 'dia de calor' aquele em que a temperatura aparente $\left(\mathrm{T}_{\mathrm{a}}\right)$ do dia excede o limiar do percentil 90 de cada um dos períodos sazonais analisados: verão (dezembro a fevereiro), outono (março a maio), inverno (junho a agosto) e primavera (setembro a novembro). Àquela OC, que iniciou em um período sazonal com término no seguinte, foi atribuída ao período em que houve o maior número de "dias de calor".

Tabela 1. Coordenadas geográficas, altitude (metros), período das séries históricas e o percentil 90 da temperatura aparente do ar $\left(\mathrm{T}_{\mathrm{a}}\right)$ dos municípios onde localizam-se as estações meteorológicas utilizadas no estudo.

\begin{tabular}{|c|c|c|c|c|c|c|c|c|}
\hline \multirow{2}{*}{ Municípios } & \multicolumn{2}{|c|}{ Coordenadas } & \multirow[b]{2}{*}{ Alt } & \multirow{2}{*}{ Período } & \multicolumn{4}{|c|}{ Percentil 90-T $\mathbf{T}_{a}\left({ }^{\circ} \mathrm{C}\right)$} \\
\hline & Lat $\left(^{\circ}\right)$ & $\operatorname{Lon}\left({ }^{\circ}\right)$ & & & Verão & Out & Inver & Prima \\
\hline Car & $-27,38$ & $-51,21$ & 964 & $1983-2015$ & 30,4 & 28,3 & 24,6 & 28,5 \\
\hline Chapecó & $-27,08$ & $-52,63$ & 687 & 1983-2015 & 32,8 & 30,7 & 26,6 & 30,7 \\
\hline Indaial & $-26,90$ & $-49,22$ & 86 & $1991-2015$ & 37,1 & 34,6 & 28,4 & 32,6 \\
\hline Lages & $-27,80$ & $-50,33$ & 936 & $1983-2015$ & 30,5 & 28,1 & 23,9 & 27,6 \\
\hline
\end{tabular}

A $T_{a}$ foi calculada utilizando a equação da regressão linear de Steadman (1984), conforme Equação 1.

$T_{a}=-1,3+0,92 \cdot T_{\text {máx }}+2,2 \cdot e_{a}$

onde $e_{a}$ é a pressão de vapor d'água do dia $(\mathrm{kPa})$, obtida com o uso da Equação 2.

$e_{a}=\frac{U R \cdot e_{S}}{100}$

onde $e_{s}$ é a pressão de saturação do vapor d'água $(\mathrm{kPa})$, estimada com o uso da expressão proposta por Tetens (Equação 3).

$e_{S}=0,6108 \cdot 10^{\frac{(7,5 . T)}{(237,3+T)}}$

As ondas de calor foram analisadas de duas formas de acordo com sua abrangência no espaço: onda de calor local (OCL) e onda de calor regional (OCR). $\mathrm{O}$ primeiro tipo analisa as ocorrências de OC de cada local, enquanto o outro tipo considera aquela em que houve coincidência de no mínimo duas datas de OC em pelo menos três das quatro estações utilizadas.
O número de ocorrências e a duração de OCL e OCR sazonal foram analisadas, em sequência, para os anos da série histórica em estudo e divididas em grupos de anos de acordo com as ocorrências dos eventos El Niño e La Niña Modoki e Canônico. Como nem sempre as datas de início e término entre as estações de uma OCR coincidem entre si, a duração do evento foi considerada pela média obtida entre cada uma das estações com registro de OC.

A cronologia de ocorrência de ambos os tipos de El Niño e de La Niña são apresentadas na Tabela 2, conforme metodologia proposta por Yeh et al. (2009), que define a ocorrência do El Niño (La Niña) Modoki quando a anomalia da temperatura da superfície do mar (ATSM) da região Niño $4\left(160^{\circ} \mathrm{E}-150^{\circ} \mathrm{W}, 5^{\circ} \mathrm{N}-5^{\circ} \mathrm{S}\right)$ está acima de $0,5^{\circ} \mathrm{C}$ (abaixo de $-0,5^{\circ} \mathrm{C}$ ) e que seja maior (menor) que a ATSM da região Niño 3 $\left(150^{\circ} \mathrm{W}-90^{\circ} \mathrm{W}, 5^{\circ} \mathrm{N}-5^{\circ} \mathrm{S}\right)$. Os eventos de El Niño (La Niña) Canônico são aqueles em que a ATSM da região Niño 3 está acima de $0,5^{\circ} \mathrm{C}$ (abaixo de $0,5^{\circ} \mathrm{C}$ ) e que seja maior (menor) do que a ATSM da região Niño 4. Quanto aos 'anos neutros', foram considerados aqueles em que não houve a ocorrência de qualquer um dos tipos de El Niño ou La Niña.

Tabela 2. Cronologia de eventos El Niño/La Niña Modoki e Canônico e de "anos neutros" durante os anos de 1982 a 2015.

\begin{tabular}{|c|c|}
\hline Eventos & Mês/ano (início) - Mês/ano (final) \\
\hline $\begin{array}{l}\text { El Niño } \\
\text { Canônico }\end{array}$ & $\begin{array}{l}\text { jun/1982-ago83; out/1986-nov/87; jun/1991-mai/92; mai/1997-mai/98; ago/2006-jan/07; } \\
\text { jun/2009-abr/10 }\end{array}$ \\
\hline $\begin{array}{l}\text { La Niña } \\
\text { Canônico }\end{array}$ & jun/1984-fev/86; abr/1988-dez/88; ago/1995-jun/96; ago/1999-jan/00; mai/2007-dez/07 \\
\hline $\begin{array}{l}\text { El Niño } \\
\text { Modoki }\end{array}$ & nov/1990-mai/91; jul/1994-mai/95; fev/2002-mar/03; jul/2004-abr/05; abr/2014-mar/15 \\
\hline La Niña & set/1983-ago/84; jul/1998-jul/99; fev/2000-mar/01; jan/2008-mai/09; set/2010-mai/11 \\
\hline
\end{tabular}


Anos neutros mar/1986-set/86; jan/1989-out/90; jun/1992-jun/1994; jul/1996-abr/97; abr/2001-jan/02; abr/2003-jun/04; mai/2005-jul/06; jun/2011-mar/14

As análises com as fases do ENOS Modoki e Canônico envolveram a primavera do ano 0 até o outono do ano +1 .

Com o número de ocorrências e duração de OCL e OCR, analisados em sequência e divididos de acordo com as fases dos dois tipos de El Niño e de La Niña, foram realizadas as seguintes análises: a) Medidas de posição e dispersão, sendo que, a média entre os valores obtidos para cada tipo de El Niño e La Niña foi comparada estatisticamente aos níveis de $5 \%$ e $1 \%$ pelo valor-p; b) Distribuição de frequência representada por histograma; c) A análise de regressão foi utilizada para indicar alterações climáticas por meio da significância do coeficiente angular de uma reta ajustada aos dados. O teste consiste em determinar o intervalo de confiança do coeficiente, e se este intervalo não incluir o valor zero, a tendência é significativa ao nível de 5\%.

\section{Resultados}

A Figura 2 apresenta o número total de ocorrências sazonal de ondas de calor e suas respectivas frequências relativas para cada município utilizado no estudo. Em Chapecó e Campos Novos ocorreu uma discreta predominância na classe de uma ocorrência de ondas de calor (37\% e $36 \%$, respectivamente) em relação a ausência de ondas de calor por período sazonal (36\% e 34\%, respectivamente). Raciocínio inverso é aplicado na análise para Indaial e Lages. A partir de duas ocorrências de ondas de calor, a frequência diminui gradativamente para todas as localidades, de forma que, quatro ocorrências foi o máximo observado de ondas de calor sazonal, representando apenas de 1\% (Campos Novos e Indaial) a 3\% (Lages) dentre todas as classes de ocorrências analisadas.

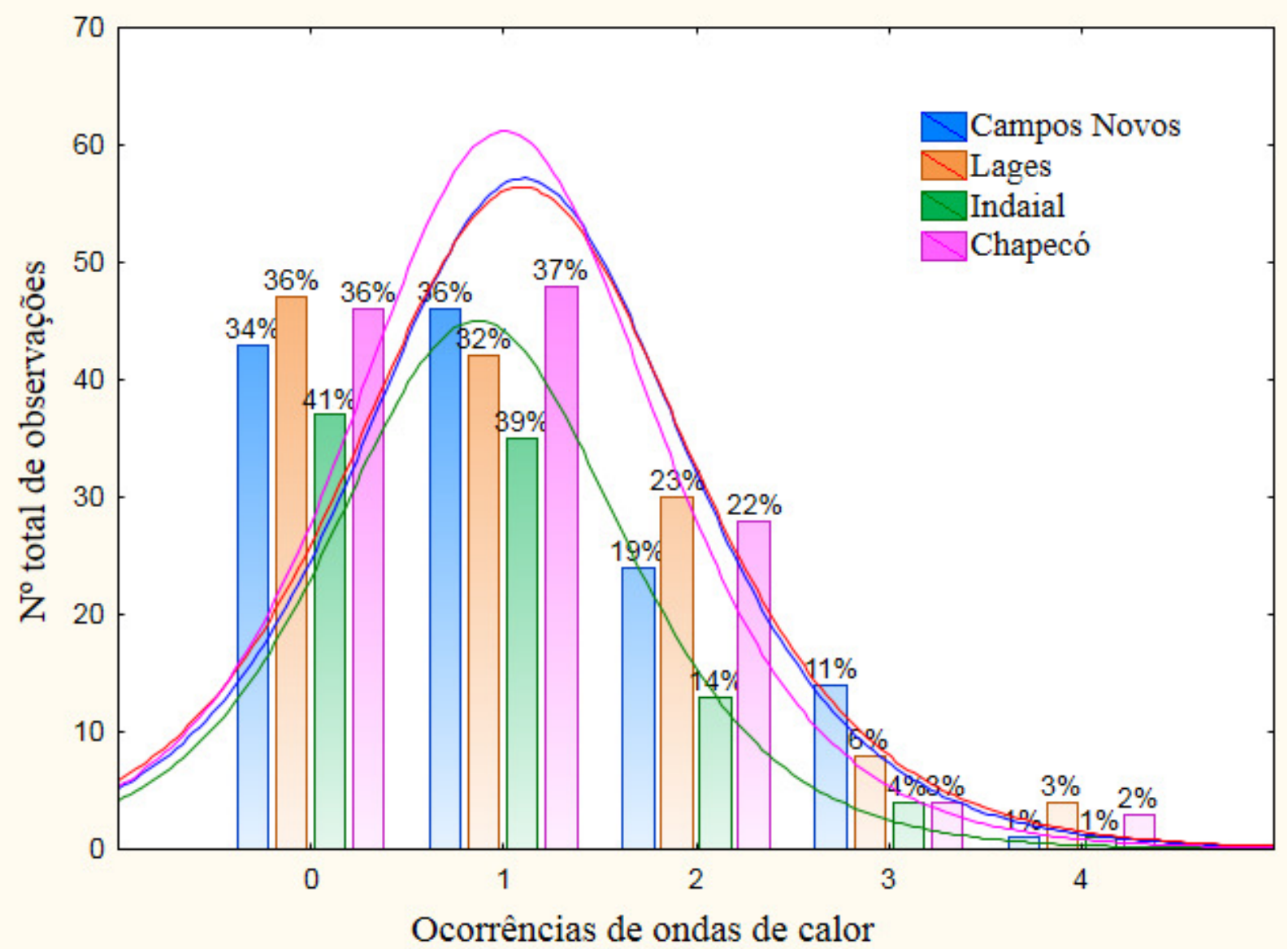

Figura 2. Número total de observações sazonais de ocorrências de ondas de calor em municípios de Santa Catarina em 1983 a 2015.

Quanto à duração das ondas de calor, a Tabela 3 mostra que os "dias de calor", durante as ondas de calor sazonal, duram em média de 7 a 8 dias (valores arredondados), não diferindo

estatisticamente entre si. Assim, pela localização geográfica dos municípios utilizados no estudo, pode-se presumir que no estado de Santa Catarina, as ondas de calor duram de 6 a 10 dias com $95 \%$ 
de confiança. Apesar disso, outono de 2002 e verão de 2014, foram períodos em que se registraram as ondas de calor de maior duração. No primeiro período, a configuração sinótica que propiciou a ocorrência do evento de 29 a 34 dias de duração, parece ter predominado da região central ao oeste do Estado, enquanto no verão de 2014, esta configuração provavelmente deve ter sido mais atuante da região serrana em direção ao litoral catarinense, em consideração pelas localizações de Lages e Indaial.

Tabela 3. Média (MD) e respectivo intervalo de confiança a 95\% da duração total sazonal de "dias de calor" durante as ondas de calor, e o percentil 90 (p 90) da duração de cada onda de calor com sua maior duração (dias) e o período de ocorrência nos municípios utilizados no estudo

\begin{tabular}{lccccc}
\hline Município & $\mathbf{- 9 5 \%}$ MD & MD $^{\mathbf{1}}$ & $\mathbf{+ 9 5 \%} \mathbf{M D}$ & Máxima/período & p 90 \\
\hline Campos Novos & 7,3 & $8,4 \mathrm{a}$ & 9,6 & $29 /$ outono-2002 & 14 \\
Lages & 5,7 & $7,2 \mathrm{a}$ & 8,7 & $27 /$ verão-2014 & 14 \\
Indaial & 6,3 & $8,2 \mathrm{a}$ & 10 & $31 /$ verão-2014 & 13 \\
Chapecó & 7,1 & $8,3 \mathrm{a}$ & 9,5 & $34 /$ outono-2002 & 14 \\
\hline
\end{tabular}

${ }^{1}$ Médias seguidas de mesma letra não diferem estatisticamente entre si ao nível de $5 \%$.

Em uma opção de critério para definir a intensidade de uma onda de calor, a Tabela 3 mostra que um evento com duração de pelo menos 14 dias (obtido pelo p 90) pode ser considerado de forte intensidade para o Estado.

Os coeficientes angulares de regressão para o número de ocorrências e duração total de 'dias de calor' durante as ondas de calor mostram tendências significativas de aumento apenas em algumas situações para a região de Lages (Tabela 4). Desde o início da década de 1980 houve um aumento de quase dois eventos de ondas de calor durante o verão de Lages, enquanto que os "dias de calor" aumentaram em torno de cinco dias no mesmo período.

Tabela 4. Coeficiente angular do número de ocorrências da série histórica e para cada período sazonal e da duração total de "dias de calor" durante as ondas de calor local, em municípios de Santa Catarina de 1983 a 2015 .

\begin{tabular}{lcccccc}
\hline Municípios & & \multicolumn{2}{c}{ Número de ocorrências } & & \multicolumn{2}{c}{$\begin{array}{c}\text { Duração } \\
\text { total }\end{array}$} \\
\hline Chapecó & Sequência & Verão & Outono & Inverno & Prima & $0,0,01$ \\
Indaial & 0,00 & 0,01 & 0,03 & 0,01 & 0,01 & 0,02 \\
Lages & 0,02 & 0,04 & 0,03 & 0,01 & 0,00 & 0,14 \\
Campos Novos & $0,02^{*}$ & $0,05^{*}$ & $0,03^{*}$ & 0,01 & 0,01 & $0,16^{* *}$ \\
\hline
\end{tabular}

* Significativo a 5\%; ** Significativo a $1 \%$

Em uma análise da abrangência espacial das ondas de calor, a Tabela 5 mostra que a duração das ondas de calor regional apresentou tendência de aumento no verão $(b=0,47)$. $O$ coeficiente obtido para o verão foi tão expressivo, que mesmo sem uma significância estatística para os demais períodos sazonais, a análise em sequência cronológica dos dados acabou sendo influenciada $(b=0,03)$. Em suma, desde meados da década de 1980, a duração das ondas de calor regional observadas no verão aumentou em torno de 4,7 dias por década. Porém, quanto ao número de ocorrências de ondas de calor regional analisadas em sequência, não houve tendência estatisticamente significativa $(b=0,03)$.

Tabela 5. Coeficiente angular da duração das ondas de calor regional da série histórica e para cada período sazonal, em municípios de Santa Catarina de 1983 a 2015.

\begin{tabular}{ccccc}
\hline Sequência & Verão & Outono & Inverno & Primavera \\
\hline $0,03^{*}$ & $0,47^{*}$ & 0,04 & 0,1 & 0,00 \\
\hline
\end{tabular}

* Significativo a $5 \%$.

Sendo o El Niño Oscilação Sul o principal mecanismo de variabilidade climática interanual, principalmente na chuva e temperatura, a Figura 3 apresenta o histograma referente ao número de ocorrências de ondas de calor da primavera (ano
0 ), verão (ano 0$)$ e outono $($ ano +1$)$ dos dois tipos de El Niño e de La Niña, bem como, dos anos sem registros desta forçante climática. A ausência, ou apenas uma ocorrência de onda de calor, durante os três períodos sazonais foi mais observada em 
anos neutros que, de certa maneira, era esperada, tendo em vista que o número de dados de anos neutros é maior, por isso tende a ser a de maior ocorrência (Figura 2).
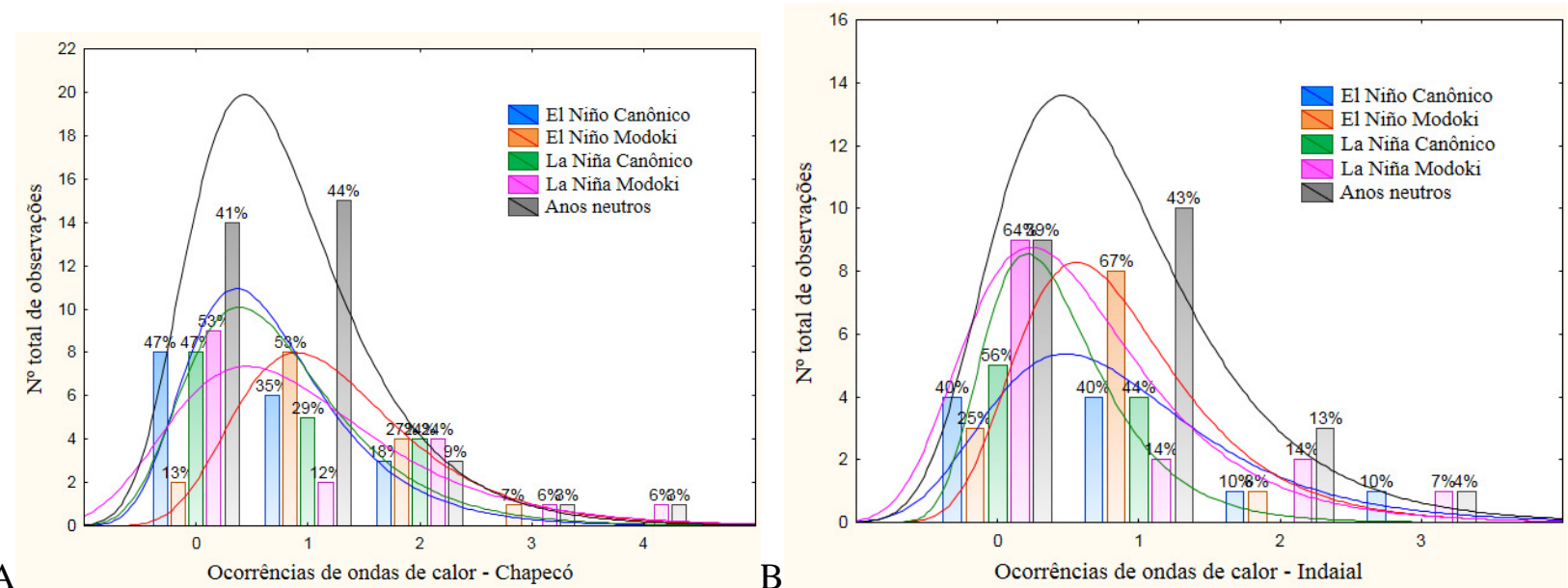

A

B

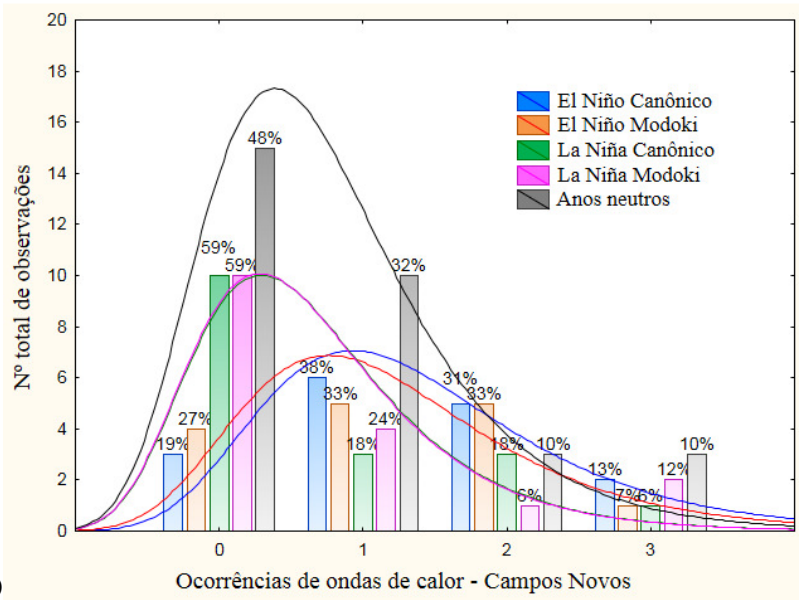

C

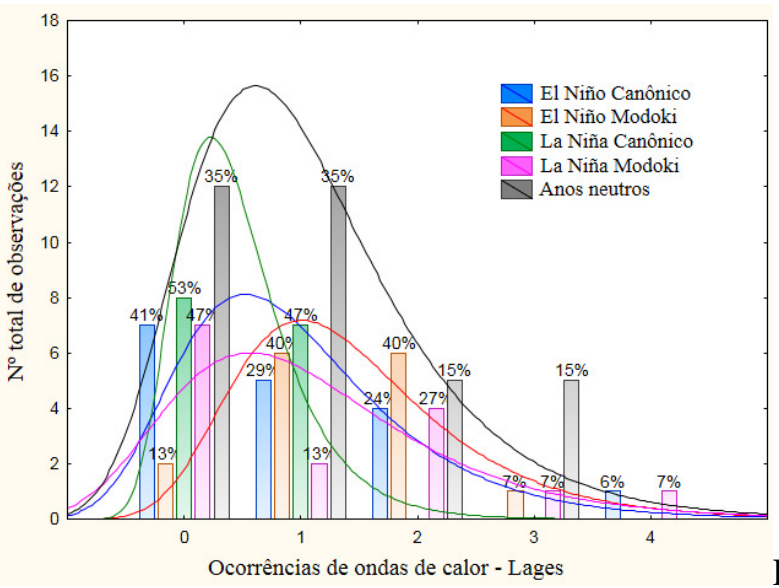

$\mathrm{D}$

Figura 3. Número total de observações de ocorrências de ondas de calor da primavera (ano 0) até o outono (ano +1) durante eventos do ENOS Modoki e Canônico e anos neutros: A. nos municípios de Chapecó, B. em Indaial, C. em Lages e D. em Campos Novos.

Porém, em uma análise de cada classe de ocorrência para cada um dos tipos de El Niño e de La Niña, isto é, sem comparações um com o outro, pode-se notar que em anos de El Niño e de La Niña Canônico há uma maior ausência de ondas de calor, e a frequência de uma até quatro ocorrências diminui gradativamente, exceto em Campos Novos para El Niño Canônico, onde a probabilidade de uma ocorrência é maior. Para La Niña Modoki a única unanimidade é a maior frequência de que não ocorram ondas de calor, visto que, para as demais classes de ocorrência há distinções entre as estações. Chama a atenção, os resultados para anos de El Niño Modoki, onde a maior probabilidade é de ocorrerem um ou dois eventos de onda de calor.

A Tabela 6 mostra que, em média, praticamente não há diferença estatisticamente significativa na duração de 'dias de calor' durante ondas de calor observadas nos dois tipos de El Niño e de La Niña, em relação aos anos neutros. A exceção é feita para Lages, onde os 'dias de calor' duram em média 4,3 dias (ao lado de Indaial, a menor duração dentre todos os valores obtidos) em anos de La Niña Canônico, enquanto em anos neutros, a duração praticamente duplica (8,3 dias). Convém lembrar que Lages foi a única localidade que teve tendência de aumento na duração de "dias de calor" desde meados da década de 1980 (Tabela 4). É plausível este achado, visto que das cinco ocorrências de La Niña Canônico analisadas, apenas uma foi registrada no século 21 (Tabela 2), isto é, no decorrer das últimas décadas diminuiu a frequência de uma forçante climática que resulta em menor duração de 'dias de calor' do que em anos sem atuação do ENOS.

Tabela 6. Média (MD) e respectivo intervalo de confiança a 95\% da duração total sazonal (primavera (ano 0) a outubro $($ ano +1$)$ ) de 'dias de calor' durante as ondas de calor nos municípios em estudo, durante eventos 
de El Niño Canônico (ENC), El Niño Modoki (ENM), La Niña Canônico (LNC), La Niña Modoki (LNM) e anos neutros (AN).

\begin{tabular}{|c|c|c|c|c|c|c|c|c|c|c|c|c|}
\hline \multirow[b]{2}{*}{ Eventos } & \multicolumn{3}{|c|}{ Campos Novos } & \multicolumn{3}{|c|}{ Indaial } & \multicolumn{3}{|c|}{ Lages } & \multicolumn{3}{|c|}{ Chapecó } \\
\hline & -95 & $\mathbf{M D}^{\mathbf{1}}$ & +95 & -95 & $\mathbf{M D}^{\mathbf{i}}$ & +95 & -95 & $\mathbf{M D}^{1}$ & +95 & -95 & $\mathbf{M D}^{\mathbf{1}}$ & +95 \\
\hline ENC & 6 & $8,0 \mathrm{a}$ & 10 & 2,1 & $9,6 a$ & 17,2 & 5,0 & $9,7 \mathrm{a}$ & 14,4 & 4,8 & $6,3 a$ & 7,9 \\
\hline ENM & 6 & $8,2 \mathrm{a}$ & 10,4 & 3,5 & $6,0 \mathrm{a}$ & 8,4 & 7,0 & $8,8 \mathrm{a}$ & 10,7 & 4,7 & $7,5 \mathrm{a}$ & 10,2 \\
\hline LNC & 4,7 & $9,6 \mathrm{a}$ & 14,4 & 1,7 & $4,0 \mathrm{a}$ & 6,2 & 2,6 & $4,3 b$ & 6,0 & 4,4 & $7,1 \mathrm{a}$ & 9,8 \\
\hline LNM & 2 & $8,6 \mathrm{a}$ & 15,0 & 1,4 & $12,2 \mathrm{a}$ & 23,0 & 5,3 & $10,2 \mathrm{a}$ & 15,1 & 5,2 & $11,1 \mathrm{a}$ & 17,1 \\
\hline AN & 4,7 & $7,5 \mathrm{a}$ & 10,2 & 2,7 & $7,1 \mathrm{a}$ & 11,4 & 5,4 & $8,3 \mathrm{a}$ & 11,2 & 5,4 & $7,6 \mathrm{a}$ & 9,9 \\
\hline
\end{tabular}

${ }^{1}$ Médias com letras iguais não diferem estatisticamente ao nível de 5\% em relação aos anos neutros (AN).

Tabela 7. Coeficiente de variação e maior duração total sazonal (primavera (ano 0 ) a outubro (ano +1)) de "dias de calor" durante ondas de calor nos municípios em estudo, durante eventos de El Niño Canônico (ENC), El Niño Modoki (ENM), La Niña Canônico (LNC), La Niña Modoki (LNM) e anos neutros (AN).

\begin{tabular}{lcccccccc}
\hline \multirow{2}{*}{ Eventos } & \multicolumn{4}{c}{ Maior duração (dias) } & \multicolumn{3}{c}{ Coeficiente de variação(\%) } \\
& CN & Indaial & Lages & Chapecó & CN & Indaial & Lages & Chapecó \\
\hline ENC & 12 & 21 & 22 & 9 & 41 & 74 & 67 & 31 \\
ENM & 15 & 13 & 15 & 17 & 40 & 53 & 34 & 60 \\
LNC & 16 & 6 & 7 & 15 & 54 & 35 & 37 & 49 \\
LNM & 20 & 26 & 22 & 23 & 82 & 71 & 57 & 64 \\
AN & 21 & 31 & 27 & 18 & 68 & 107 & 77 & 63 \\
\hline
\end{tabular}

\section{Discussão}

Mesmo que as localidades e o período em estudo sejam iguais, a frequência de ocorrência de ondas de calor pode diferir se utilizados outros critérios para sua definição. Apesar de não haver uma definição padrão para ondas de calor, é comumente atribuído o evento com uma sequência de 'dias de calor' ou com temperaturas e umidade do ar muito elevadas para o local e época do ano. Por exemplo, Robinson (2001) descreveu uma onda de calor como um extenso período incomum de alto estresse por calor relacionado às condições meteorológicas que causa temporariamente modificações nos hábitos de vida e problemas na saúde de comunidades. Assim, a análise dos elementos meteorológicos deve sempre incluir a avaliação da sensação de calor humana. Limites apropriados devem ser estabelecidos para esta análise combinada, considerando valores extremos de temperatura do ar. Os limites também devem ser relacionados com a variabilidade climática da região em estudo. Por estes apontamentos que se optou pela utilização da temperatura aparente e o percentil 90 para determinar as ocorrências de ondas de calor.

Alves et al. (2016), utilizando uma série histórica maior de dados meteorológicos, bem como, de estações distribuídas por Santa Catarina, atestam a onda de calor durante o verão de 2014 como a mais duradoura e atuante no Vale do Itajaí e áreas adjacentes, mesmo adotando uma metodologia diferente para identificação de ondas de calor em relação àquela adotada no presente estudo. Em uma análise do padrão atmosférico, os autores acrescentam que o referido evento de onda de calor, foi ocasionado por um bloqueio atmosférico no oceano Pacífico Sul, que alterou o padrão de escoamento de vento e a trajetória das frentes frias que, chegando ao máximo no sul do Rio Grande do Sul, eram desviadas para o oceano Atlântico. Para elucidar, Ambrizzi et al. (2009) informam que um bloqueio atmosférico corresponde a uma anomalia persistente de alta pressão, que tem o deslocamento meridional como característica e em relação às trajetórias normais zonais das perturbações atmosféricas nos subtrópicos e latitudes médias.

Esta tendência nas características das ondas de calor também é observada em outras regiões do planeta. Unal et al. (2013) mostraram que o número de dias de calor, ondas de calor e sua duração aumentou entre 1965 a 2006, na região oeste da Turquia. Adicionalmente, a taxa de mudança foi maior durante a última década e eventos extremos foram frequentemente observados após 1998. Encontraram correlações de dias de calor com ocorrências de incêndios e temperatura superficial do Mar Mediterrâneo e do Mar Negro.

Em grande parte da China, Ding et al. (2009) mostraram que a frequência de ondas de calor foi alta durante os anos de 1960 e 1970, baixas nos anos de 1980, e a partir dos anos de 1990, uma notável tendência de aumento com fortes variações interanuais. Acrescentam que essas mudanças estiveram principalmente relacionadas aos dias chuvosos e padrão de circulação atmosférica nas escalas interanual e interdecadal. Para a região de Lages, Minuzzi \& Lopez (2014) encontraram tendência de aumento 
no número de dias chuvosos, que pode sugerir aumento da temperatura e umidade do ar, mas uma conclusão mais precisa, seria viável se houvesse uma análise dos tipos de chuvas que resultaram nesta tendência.

Somado a isto, pode-se acrescentar as variabilidades na temperatura do ar, como apresentado no estudo de DeGaetano \& Allen (2002), que encontraram aumento nas ocorrências de temperaturas extremas em todo o território americano durante 1960 a 1996 , com as maiores tendências sendo observadas em áreas urbanas.

Se considerarmos a temperatura máxima, seria incoerente de acordo com Minuzzi (2010), que encontrou tendência de diminuição em Lages, numa análise de 1955 a 2008. Porém, o autor identificou pontos de mudança climática mensal na referida série histórica em torno do ano de 1996 e, após este período da mudança, não houve tendência significativa.

Della-Marta et al. (2007) destacaram que a duração das ondas de calor no verão boreal aumentou de 1,4 a 3 dias por evento e a frequência de 'dias de calor' triplicou na Europa Ocidental nos anos recentes comparados com o início do século 20.

Acrescentando eventuais efeitos das ondas de calor, $\mathrm{Xu}$ et al. (2012), em estudo observacional, também encontraram uma tendência de aumento nas ondas de calor que estiveram associadas a diminuição no crescimento da vegetação no norte da China. Bauweraerts et al. (2014) concluíram que as ondas de calor produziram mais estresse em duas espécies de árvores do que a mesma quantidade de calor aplicada uniformemente. Os benefícios de um aumento do $\mathrm{CO}_{2}$ pareciam diminuir com o aumento na intensidade das ondas de calor. Assim, quanto ao desempenho em cenários climáticos previstos, a intensidade e duração dos eventos de calor devem ser mais importantes do que a magnitude do aumento médio da temperatura.

Quanto ao ENOS, Yeh et al. (2009) estudaram a razão entre a frequência de El Niño Modoki e Canônico no clima presente e futuro. A razão entre ambos os tipos de El Niño foi maior no futuro do que no presente, associando assim o aumento na frequência de EN Modoki às mudanças climáticas. Lee \& McPhanden (2010) chamam a atenção que o aumento na frequência de El Niño Modoki projetado por Yeh et al. (2009) pode ser em razão do aumento gradual nas anomalias positivas de TSM no Pacífico Central em anos de El Niño, que não tem ocorrido em anos neutros ou de La Niña.

\section{Conclusão}

O número de "dias de calor" durante as ondas de calor local dura entre seis e 10 dias, ocorrendo, com mais frequência, apenas uma vez a cada estação do ano.

Apenas na região de Lages houve tendência climática significativa, com aumento no número de ocorrências de ondas de calor e na duração de "dias de calor".

A duração das ondas de calor com abrangência em grande escala, aumentou em torno de 4,7 dias/década no verão em Santa Catarina.

El Niño e La Niña Modoki ou Canônico não influenciam na duração de "dias de calor" durante ondas de calor, exceto em Lages, durante eventos de La Niña Modoki.

\section{Agradecimentos}

Os autores agradecem à Universidade Federal de Santa Catarina pela concessão de bolsa de estudo para a segunda autora.

\section{Referências}

ALVES, M. P. A., VANZ, A.; CRUZ, G. S.; MARTARELLO, K. C. B. K.; MONTEIRO, A. N.; MINZINSKI, J. 2016. Caracterização da forte onda de calor de 2014 em Santa Catarina. Ciência e Natura, v. 38, n. 1, p. 309-325.

AMBRIZZI, $\quad$ T.; $\quad$ MARQUES, R.; NASCIMENTO, E. 2009. Bloqueios Atmosféricos. In: CAVALCANTI, I. F. de A., et al. (org.), Tempo e clima no Brasil, São Paulo: Oficina de Textos. pp. 279-296.

BAUWERAERTS, I., AMEYE, M.; WERTIN, T. M.; McGUIRE, M. A.; TESKEY, R. O.; STEPPE, K. 2014. Water availability is the decisive factor for the growth of two tree species in the occurrence of consecutive heat waves. Agricultural and Forest Meteorology, v. 189-190, p. 19-29.

DELLA-MARTA, P. M.; LUTERBACHER, J.; VON WEISSENFLUH, H.; XOPLAKI, E.; BRUNET, M.; WANNER, H. 2007. Summer heat waves over western Europe 1880-2003, their relationship to large scale forcings and predictability. Climate Dynamics, v. 59, p. 251275.

DIFFENBAUGH, N. S.; PAL, J. S.; TRAPP, R. J.; GIORGI, F. 2005. Fine scale processes regulate the response of extreme events to global climate change. Proc. Natl. Acad. Sci. USA, v. 102, n. 44, p. 15774-15778. 
DING, T.; QIAN, W.; YAN, Z. 2010. Changes in hot days and heat waves in China during 19612007. International Journal of Climatology, v. 30, p. $1452-1462$.

GRIMM, A. M. 2009. Clima da região sul do Brasil. In: CAVALCANTI, I. F. de A. et al. (org.), Tempo e clima no Brasil, São Paulo: Oficina de Textos, pp. 259-275.

LARKIN, N. K.; HARRISON, D. E. 2005. Global seasonal temperature and precipitation anomalies during El Niño autumn and winter. Geophysical Research Letters, v. 32, n. 16, p. L16705.

LEE, T.; MCPHADEN, M. J. 2010. Increasing intensity of El Niño in the central equatorial Pacific. Geophysical Research Letters, v. 37, L14603.

MINUZZI, R. B.; LOPEZ, F. Z. 2014. Variabilidade de índices de chuva nos estados de Santa Catarina e Rio Grande do Sul. Bioscience Journal, v. 30, n. 3, p. 697-706.

MINUZZI, R. B. 2010. Tendências na variabilidade climática de Santa Catarina, Brasil.
Revista Brasileira de Engenharia Agrícola e Ambiental, v. 14, n. 12, p. 1288-1293.

ROBISON, P.J. 2001. On the definition of heat waves. Journal Applied Meteorology, v. 40, p. 762-775.

ROSS, T., LOTT, N. 2003. A climatology of 1980-2003 extreme weather and climate events. National Climatic Data Center Tech. Rep. 200301, 14p.

UNAL; Y. S.; TAN; E.; MENTES, S. S. 2013. Summer heat waves over western Turkey between 1965 and 2006. Theoretical Application Climatology, v. 112, p. 339-350.

XU, X.; PIAO, S.; WANG, X.; CHEN, A.; CIAO, P.; MYNENI, R. B. 2012. Spatio-temporal patterns of the area experiencing negative vegetation growth anomalies in China over the last three decades. Environmental Research. Letters, v. 7, n. 3, p. 1-9.

YEH, S.; KUG, J. S.; DEWITTE, B.; KWON, M. H.; KIRTMAN, B. P.; JIN, F. F. 2009. El Niño in a changing climate. Nature, v. 461, p. 511-514. 